\title{
Penrose limits, worldvolume fluxes, and supersymmetry
}

\author{
David Mateos* \\ DAMTP, Centre for Mathematical Sciences, University of Cambridge, Wilberforce Road, Cambridge CB3 OWA, United Kingdom
}

(Received 22 July 2002; published 29 October 2002)

\begin{abstract}
It is noted that a finite Penrose limit for brane probes with nonzero worldvolume fluxes does not generically exist; this is closely related to the observation by Blau and co-workers that for a brane probe the Penrose limit is equivalent to an infinite-tension limit. It is shown that when the limit exists, however, the number of supersymmetries preserved by the probe does not decrease.
\end{abstract}

DOI: 10.1103/PhysRevD.66.086003

PACS number(s): 11.25. $-\mathrm{w}, 04.65 .+\mathrm{e}, 11.30 . \mathrm{Pb}$

\section{INTRODUCTION}

Three crucial features of the Penrose limit [1] of a supergravity solution [2] along a null geodesic are (i) that a finite limit always exists, (ii) that the resulting configuration is also a solution of the supergravity field equations, and (iii) that the number of supersymmetries of the initial solution does not decrease in the limit [3]. In fact, the precise rescalings of the supergravity fields involved in taking the limit are dictated by demanding that conditions (i) and (ii) be satisfied.

Brane probes in supergravity spacetimes [4] are an essential tool for understanding both spacetime geometry and gauge theory physics [5]. One purpose of this paper is to observe that property (i) does not extend to solutions of the equations of motion of a brane probe with generic non-zero worldvolume gauge fields; this will be illustrated with a simple example. This result is closely related to the fact that the Penrose limit for the supergravity background can be reinterpreted as an infinite-tension limit for the probe $[3,6]$; we will return to this in Sec. V.

In the context of the AdS/CFT (conformal field theory) correspondence, two examples of brane probes in $\mathrm{AdS}_{5}$ $\times S^{5}$ with non-zero worldvolume gauge fields are provided by the "defect" D5-brane [7] and the "baryonic" D5-brane [8]. These are especially relevant to the present discussion because, as we will explain, they share a non-generic feature that allows the general arguments presented here to be circumvented and thus a finite limit to be defined.

Property (ii) does extend to brane probe solutions $[3,6]$ provided of course a well-defined Penrose limit exists. The introduction of the probe generically preserves only a fraction (possibly none) of the supersymmetries of the supergravity background. It has been tacitly assumed in the literature that the number of supersymmetries preserved by the probe does not decrease in the Penrose limit, that is, that property (iii) also extends to brane probes. Since to our knowledge no proof of this has been presented, we provide a simple one here.

\section{WORLDVOLUME FLUXES}

In the neighborhood of a segment of a null geodesic with no conjugate points the metric may be written as $[1-3]$

\footnotetext{
*Email address: D.Mateos@damtp.cam.ac.uk
}

$$
g=d V\left(d U+\alpha d V+\beta_{i} d Y^{i}\right)+C_{i j} d Y^{i} d Y^{j},
$$

where $\alpha, \beta_{i}$ and $C_{i j}$ are functions of all the coordinates. The geodesic lies at $V=Y^{i}=0$ and is affinely parametrized by $U$. Let us introduce new rescaled coordinates

$$
U=u, \quad V=\Omega^{2} v, \quad Y^{i}=\Omega y^{i},
$$

where $\Omega$ is a positive real constant, and set $g_{\Omega}=\Omega^{-2} g$. The Penrose limit of $g$ is obtained by computing the limit

$$
\bar{g} \equiv \lim _{\Omega \rightarrow 0} g_{\Omega}
$$

while keeping $u, v$ and $y^{i}$ fixed. Similarly, if $B_{k-1}$ is a supergravity gauge potential subject to the gauge transformation

$$
\delta B_{k-1}=d \Lambda_{k-2}
$$

and $H_{k}=d B_{k-1}$ is its gauge-invariant $k$-form field strength [9], then we set

$$
H_{\Omega}=\Omega^{-k+1} H
$$

and define the Penrose limit of $H$ as $\bar{H} \equiv \lim _{\Omega \rightarrow 0} H_{\Omega}$, again keeping $u, v$ and $y^{i}$ fixed. It is easy to see that these limits are finite [1-3].

In the presence of branes there are in general additional gauge potentials $A_{k-1}$ (for certain values of $k$ ) that describe degrees of freedom localized on the worldvolumes of the branes. Examples of these include a one-form gauge potential in the case of D-branes and a two-form gauge potential in the case of the M5-brane. Their gauge-invariant field strengths $\mathcal{F}_{k}$ involve the supergravity gauge potentials through combinations of the form

$$
\mathcal{F}_{k}=F_{k}+B_{k}^{\star},
$$

where $F_{k}=d A_{k-1}$ and " $\star$ " denotes the pull-back to the worldvolume. The reason is that in the presence of branes the theory is not invariant under the gauge transformations (4) alone but in combination with [10] $\delta A_{k-2}=-\Lambda_{k-2}^{\star}$. In the case of D-branes $B_{2}$ is the Neveu-Schwarz two-form, whereas for the M5-brane $B_{3}$ is the three-form potential of 11-dimensional supergravity.

Equation (6) has crucial implications for the existence of finite Penrose limits for brane probes because it means that, 
in order to extend the definition of the limit to the worldvolume gauge fields in a gauge-invariant manner, we must define $F_{\Omega}=\Omega^{-k} F$ and $\bar{F} \equiv \lim _{\Omega \rightarrow 0} F_{\Omega}$. It then follows that $\mathcal{F}_{\Omega}=\Omega^{-k} \mathcal{F}$ and

$$
\overline{\mathcal{F}} \equiv \lim _{\Omega \rightarrow 0} \mathcal{F}_{\Omega}
$$

We thus see that gauge invariance forces the $k$-form field strength $\mathcal{F}_{k}$ to be rescaled with one extra power of $\Omega^{-1}$ as compared to a supergravity field strength of the same rank.

To see the consequences of this, consider a null geodesic that intersects, or is contained within [11], the worldvolume of the brane. For generic non-zero values of $F_{k}$ and $B_{k}$ the components $\mathcal{F}_{U i_{1} \ldots i_{k-1}}$ of the worldvolume flux $\mathcal{F}_{k}$ will be non-zero in a neighborhood of the geodesic. Since these components lead to terms that scale as $\Omega^{k-1}$, the limit (7) diverges. Needless to say, one may choose to gauge away the analogous components of $B_{k}$ or of $F_{k}$, but the gaugeinvariant field strength $\mathcal{F}_{k}$ will remain unchanged.

Since $\mathcal{F}_{k}$ has support solely on the worldvolume of the brane, it is clear that the presence of the probe does not affect the Penrose limit along a null geodesic that lies entirely ouside the worldvolume. In fact, since only a region infinitesimally close to the geodesic (which is magnified by an infinite amount) survives the limit, the brane just disappears from the resulting spacetime.

The obstruction to the existence of a finite Penrose limit is not visible in an effective description in which the brane is replaced by its backreaction on spacetime, that is, by some supergravity solution, because in this description the worldvolume flux $\mathcal{F}$ is ignored [13]. Moreover, most of these supergravity solutions are typically singular at the location of the putative brane, so their Penrose limits along geodesics that intersect the brane are not usually considered.

The brane probe action is homogeneous under the rescalings above $[3,6]$; for example, the action for a $\mathrm{D} p$-brane in a supergravity background

$$
S_{p}=-\int e^{-\phi} \sqrt{-\operatorname{det}(g+\mathcal{F})}+\int e^{\mathcal{F}} \wedge C
$$

is homogeneous [12] of degree $-(p+1)$. This property ensures that solutions of the brane equations of motion in a supergravity spacetime are mapped by the Penrose limit to new solutions in the resulting spacetime, provided of course that the limit for the probe exists.

Brane probes play an important role in the context of the AdS/CFT correspondence. Two examples in an $\mathrm{AdS}_{5} \times \mathrm{S}^{5}$ background are the defect D5-brane [7] and the baryonic D5-brane [8]. These are especially relevant to the present discussion because the worldvolume flux $\mathcal{F}$ is non-zero in both cases, yet finite Penrose limits for the defect brane and for the baryonic brane were found in [14] and [15], respectively [16]. The non-generic feature common to these two cases that allows the arguments above to be circumvented is that the overall scale of $\mathcal{F}$ is arbitrary; more precisely, there is an entire family of solutions parametrized by the magnitude of $\mathcal{F}$ [17]. This implies that $\mathcal{F}$ can be rescaled with an arbitrary power of $\Omega$ that, in particular, can be appropriately chosen in order to make the limit (7) finite; in effect, this means that one does not take the Penrose limit of a fixed solution, but instead focuses on an $\Omega$-dependent member of the family as $\Omega$ is scaled to zero. It is this additional freedom that allowed a finite limiting result to be obtained in $[14,15]$.

\section{AN EXAMPLE}

A simple example in which the freedom discussed above to rescale the worldvolume flux at will does not occur is provided by the supersymmetric $\mathrm{D} 3 / \overline{\mathrm{D} 3}$ system. This consists of a stack of $N$ infinite flat D3-branes separated by some distance from a parallel stack of $\bar{N}$ anti-D3-branes. On the worldvolumes of both groups of branes there are constant (Abelian) electric fields $\mathcal{E}$ and $\mathcal{E}^{\prime}$ (aligned with each other) and constant magnetic fields $\mathcal{B}$ and $\mathcal{B}^{\prime}$ (also aligned with each other but orthogonal to the electric fields). For generic values of these worldvolume fluxes this system is unstable: there is a long-distance force between the two groups of branes [18], and for sufficiently small separation an openstring tachyonic mode develops. However, if $\mathcal{E}=\mathcal{E}^{\prime}= \pm 1$ and $\mathcal{B}$ and $\mathcal{B}^{\prime}$ are non-zero [19] and have opposite signs, then the whole system preserves $1 / 4$ supersymmetry (hence the force vanishes [20,21] and no tachyonic instability appears $[22,23])$. This follows from the fact that this configuration is T-dual to the supersymmetric D2/D2 system $[24,22]$, whose supersymmetry can in turn be understood from its origin as a particular limit of the D2-brane supertube [25].

If $N=1$ and $g_{s} \bar{N} \gg 1$, where $g_{s}$ is the string coupling constant, then the appropriate description of this system is in terms of a D3-brane probe in the supergravity background created by the $\bar{N} \overline{\mathrm{D} 3}$-branes. In this case the condition $|\mathcal{E}|$ $=1$ arises from the requirement that the equations of motion derived from the action (8) with $p=3$ are solved by a static D3-brane probe with worldvolume electric and magnetic fields [20]. (Note that although this condition happens to imply preservation of $1 / 4$ supersymmetry, this need not be imposed a priori.) Since the value of the worldvolume electric field on the probe is now fixed, it follows from the arguments in the previous section that the Penrose limit along generic geodesics that intersect (or are contained within) the D3-brane leads to a divergent result for the worldvolume field strength.

\section{SUPERSYMMETRY}

The Penrose limit of a supergravity solution possesses at least as many supersymmetries as the original solution [3]. The essence of the argument is as follows. For each Killing spinor $\epsilon$ of the initial solution there exists a real constant $\beta$ such that the limit $\bar{\epsilon} \equiv \lim _{\Omega \rightarrow 0} \Omega^{\beta} \epsilon$ is finite. The linearity of the Killing spinor equations then implies that $\bar{\epsilon}$ is a Killing spinor of the resulting supergravity solution. The argument is completed by showing that it is possible to choose the initial basis of Killing spinors in such a way that the limiting ones are linearly independent.

The supersymmetries of a supergravity solution that are 
left unbroken by the introduction of a brane probe are those generated by spacetime Killing spinors $\epsilon$ that satisfy [26]

$$
\Gamma \epsilon=\epsilon,
$$

where $\Gamma$ is the matrix appearing in the kappa-symmetry transformations of the brane worldvolume fermions; by construction it satisfies $\Gamma^{2}=1$. Equation (9) may be regarded as the worldvolume analogue of the background Killing spinor equations. The relevant observation for our purpose is that Eq. (9) is linear and that both sides are homogeneous of the same degree under the rescalings involved in the Penrose limit; we show this explicitly below for D-branes. It follows that if a subset of the background Killing spinors $\left\{\epsilon_{i}\right\}$ verify Eq. (9) then so do their Penrose limits $\left\{\overline{\boldsymbol{\epsilon}_{i}}\right\}$, and therefore the resulting probe will preserve at least as many supersymmetries as the original one. Of course, just like in certain cases [27] the number of supersymmetries of the background may actually increase in the limit, so may the number of supersymmetries preseved by the brane $[14,15]$.

The kappa-symmetry matrix for $\mathrm{D} p$-branes in a general supergravity background can be found in $[28,29]$; since the formulas differ slightly in the type IIA and type IIB cases, we focus here on type IIB D $p$-branes for concreteness. In this case $\Gamma$ is defined by the equation

$$
\Gamma d \sigma^{0} \wedge \ldots \wedge d \sigma^{p}=\frac{1}{\sqrt{-\operatorname{det}(g+\mathcal{F})}} \sum_{n} \Gamma_{(2 n)}^{\star} K^{n} I \wedge e^{\mathcal{F}},
$$

where $\left\{\sigma^{0}, \ldots, \sigma^{p}\right\}$ are worldvolume coordinates in which the determinant on the right-hand side of Eq. (9) is calculated. $\Gamma_{(n)}^{\star}$ is the pull-back to the worldvolume of the spacetime matrix-valued $n$-form

$$
\Gamma_{(n)}=\frac{1}{n !} \Gamma_{a_{1} \ldots a_{n}} e^{a_{1}} \wedge \ldots \wedge e^{a_{n}},
$$

where $\left\{\Gamma_{a}\right\}$ are ten tangent-space constant Dirac matrices and $\left\{e^{a}\right\}$ is a basis of orthonormal one-forms for the spacetime metric, that is, $g=\eta_{a b} e^{a} e^{b} . K$ and $I$ are linear operators that act on chiral complex spinors of type IIB supergravity as $K \psi=\psi^{*}$ and $I \psi=-i \psi$. Finally, it must be understood that only the form of degree $p+1$ is selected on the right-hand side of Eq. (10).

The conformal rescaling of the background metric involved in the Penrose limit implies that the orthonormal oneforms are rescaled as $e_{\Omega}^{a}=\Omega^{-1} e^{a}$. This, together with the rescaling of $\mathcal{F}$, implies that $\Gamma_{\Omega}=\Gamma$. It then follows that both sides of Eq. (9) are homogeneous of the same degree $\beta$.

\section{DISCUSSION}

In the preceding discussion the tension of the brane probe was set to unity, and therefore implicitly kept fixed in all the rescalings involved in the Penrose limit. It was shown in $[3,6]$, however, that the Penrose limit of the supergravity background can be reinterpreted as an infinite-tension limit for the probe. Let us see the connection between this result and the definition of the Penrose limit that we have adopted. For concreteness, we focus again on a $\mathrm{D} p$-brane. If we reinstate $\alpha^{\prime}$ then the gauge-invariant field strength (6) becomes

$$
\mathcal{F}=\alpha^{\prime} F+B^{\star} .
$$

In addition, the action (8) acquires an overall factor of $\left(\alpha^{\prime}\right)^{-(p+1) / 2}$, and therefore it satisfies [3,6]

$$
S_{p}\left[\Psi_{\Omega}, F ; \alpha^{\prime}\right]=S_{p}\left[\Psi, F ; \tilde{\alpha}^{\prime}\right]
$$

where $\Psi$ collectively denotes all the background fields and $\tilde{\alpha}^{\prime}=\Omega^{2} \alpha^{\prime}$. We thus see that the Penrose limit $\Omega \rightarrow 0$ for the background spacetime translates into an infinite-tension limit $\tilde{\alpha}^{\prime} \rightarrow 0$ for the brane in the original background. On the other hand, if we now rewrite Eq. (13) as

$$
S_{p}\left[\Psi_{\Omega}, F ; \Omega^{-2} \alpha^{\prime}\right]=S_{p}\left[\Psi, F ; \alpha^{\prime}\right]
$$

then we may reinterpret it as saying that the $\mathrm{D} p$-brane action is homogeneous (of degree zero) if the background fields are rescaled as above, $F$ is kept fixed and $\alpha^{\prime}$ is rescaled as $\alpha_{\Omega}^{\prime}$ $=\Omega^{-2} \alpha^{\prime}$. Note that this definition of the limit is consistent with gauge invariance because both terms on the right-hand side of Eq. (12) are rescaled in the same way. In fact, as far as the Penrose limit of classical solutions of the action (8) is concerned, this definition and that of Sec. II always yield physically equivalent results (in particular, they both fail to exist in the same cases). The reason is that, except for the overall factor in the action (8), $\alpha^{\prime}$ and $F$ do not occur separately but only in the combination $\alpha^{\prime} F$, which in both cases is rescaled in the same way. The different overall scalings (degrees of homogeneity) of the action in the two cases could make a difference when this is inserted in some path integral, but they do not make a difference at the classical level.

In this paper we have focused on the obstructions to the existence of the Penrose limit associated with the presence of worldvolume fluxes. In some cases, however, the existence of a well-defined limit for the brane embedding itself is not a trivial issue [15]. This is consistent with the fact that the embedding is specified by worldvolume scalar fields and that these may be dual to worldvolume gauge fields in certain cases. One example of this is provided by the type IIA D2brane: its worldvolume one-form potential is equivalent to a periodically identified scalar field which, after the reinterpretation of the D2-brane as an M-theory membrane, specifies the position of the latter along the M-theory circle [29]. It follows that any obstacles to the existence of the Penrose limit due to the D2-brane gauge field must be reinterpretable in terms of the M2-brane embedding.

\section{ACKNOWLEDGMENTS}

It is a pleasure to thank José Figueroa-O'Farrill, Chris Hull, and Selena Ng for helpful discussions and comments on the manuscript. This work was supported by PPARC. 
[1] R. Penrose, in Differential Geometry and Relativity (Reidel, Dordrecht, 1976), p. 271.

[2] R. Güven, Phys. Lett. B 482, 255 (2000).

[3] M. Blau, J. Figueroa-O'Farrill, and G. Papadopoulos, "Penrose Limits, Supergravity and Brane Dynamics," hep-th/0202111.

[4] Brane probes are branes whose backreaction on spacetime can be neglected.

[5] See, for example, the excellent review by C.V. Johnson, "DBrane Primer," hep-th/0007170.

[6] M. Blau, J. Figueroa-O'Farrill, C. Hull, and G. Papadopoulos, Class. Quantum Grav. 19, L87 (2002).

[7] A. Karch and L. Randall, J. High Energy Phys. 06, 063 (2001).

[8] E. Witten, J. High Energy Phys. 07, 006 (1998).

[9] In general these gauge transformations, and hence the expressions for the gauge-invariant field strengths, are more complicated; for example, for type II supergravities see M.B. Green, C.M. Hull, and P.K. Townsend, Phys. Lett. B 382, 65 (1996). The conclusions we derive here are, however, unaffected.

[10] In addition, the theory is also invariant under $\delta B_{k}=0$, $\delta A_{k-1}=d \lambda_{k-2}$.

[11] Or that becomes arbitrarily close to the brane; for a discussion of this case see [15].

[12] The dilaton is not rescaled in the Penrose limit.

[13] Except perhaps as a source for spacetime fields.

[14] K. Skenderis and M. Taylor, J. High Energy Phys. 06, 025 (2002).

[15] D. Mateos and S. Ng, J. High Energy Phys. 08, 005 (2002).

[16] The Penrose limit of a particular case of the defect brane with zero worldvolume flux was previously studied in P. Lee and J.
Park, "Open Strings in PP-Wave Background from Defect Conformal Field Theory," hep-th/0203257. The Penrose limit of the baryonic brane was studied simultaneously with [15] in S. Seki, Phys. Lett. B 542, 165 (2002), but in this reference the worldvolume flux was not considered.

[17] The precise way in which the brane is embedded depends on the magnitude of $\mathcal{F}$.

[18] This force may be attractive or repulsive, depending on the values and signs of the electromagnetic fields; in the particular case in which they all vanish the force is attractive.

[19] This ensures that the electric fields are subcritical.

[20] R. Emparan, D. Mateos, and P.K. Townsend, J. High Energy Phys. 07, 011 (2001).

[21] D. Bak and K. Lee, Phys. Lett. B 509, 168 (2001).

[22] D. Mateos, S. Ng, and P.K. Townsend, J. High Energy Phys. 03, 016 (2002).

[23] D. Bak and N. Ohta, Phys. Lett. B 527, 131 (2002).

[24] D. Bak and A. Karch, Nucl. Phys. B626, 165 (2002).

[25] D. Mateos and P.K. Townsend, Phys. Rev. Lett. 87, 011602 (2001).

[26] See, for example, E. Bergshoeff, R. Kallosh, T. Ortín, and G. Papadopoulos, Nucl. Phys. B502, 149 (1997).

[27] N. Itzhaki, I.R. Klebanov, and S. Mukhi, J. High Energy Phys. 03, 048 (2002); J. Gomis, and H. Ooguri, Nucl. Phys. B635, 106 (2002).

[28] M. Cederwall, A. von Gussich, B.E.W. Nilsson, P. Sundell, and A. Westerberg, Nucl. Phys. B490, 179 (1997).

[29] E. Bergshoeff and P.K. Townsend, Nucl. Phys. B490, 145 (1997). 